\title{
THE OBSERVATION OF 10-50 KeV SOLAR FLARE X-RAYS \\ BY THE OGO SATELLITES AND THEIR CORRELATION WITH SOLAR RADIO AND ENERGETIC PARTICLE EMISSION
}

\author{
R. L. Arnoldy, S. R. KANE and J.R. WincKleR \\ (School of Physics and Astronomy, University of Minnesota, \\ Minneapolis, Minn., U.S.A.)
}

\begin{abstract}
A BSTRACT
More than 70 cases have been observed of energetic solar flare X-ray bursts by large ionization chambers on the OGO satellites in space. The ionization chambers have an energy range between 10 and $50 \mathrm{KeV}$ for $\mathrm{X}$-rays and are also sensitive to solar protons and electrons. A study has been made of the X-ray microwave relationship, and it is found that the total energy released in the form of X-rays between 10 and $50 \mathrm{KeV}$ is approximately proportional to the peak or total energy simultaneously released in the form of microwave emission. For a given burst the rise time, decay time and total duration are similar for the $10-50 \mathrm{KeV} \mathrm{X}$-rays and the 3 to $10 \mathrm{~cm}$ radio emission. Roughly exponential decay phases are observed for both emissions with time constants between 1 and 10 min. All 3 or $10 \mathrm{~cm}$ radio bursts with peak intensity greater than 80 solar flux units are accompanied by an X-ray burst greater than $3 \times 10^{-7} \mathrm{ergs} \mathrm{cm}^{-2} \mathrm{sec}^{-1}$ peak intensity. The probability of detecting such $\mathrm{X}$-ray events is low unless the radio spectrum extends into the centimetric range of wavelengths. The best correlation between $\mathrm{cm}-\lambda$ and energetic $\mathrm{X}$-rays is observed for the first event in a flare. Subsequent structure and second bursts may not correspond even when the radio emission is rich in the microwave component. The mechanism for the energetic X-rays is shown to be bremsstrahlung probably of fast electrons on a cooler plasma. If the radio emission is assumed to be synchrotron radiation then a relationship is developed between density and magnetic field which meets the observed quantitative results. One finds, on the average, that $5 \times 10^{-54}$ joules $\mathrm{m}^{-2}(\mathrm{CPS})^{-1}$ of microwave energy at the Earth are required per electron at the Sun to provide the radio emission for the various events.

A strong correlation between interplanetary solar flare electrons observed by satellite and X-ray bursts is shown to exist. This correlation is weak for solar proton events. One may infer a strong propagation asymmetry for solar flare electrons along the spiral interplanetary magnetic field.
\end{abstract}

\section{Introduction}

Since September 1964 ionization chambers flown in space have detected numerous solar flare X-ray bursts in the energy range from 10 to $50 \mathrm{keV}$, originating in solar flare disturbances. In the present paper we shall present the most recent summary of these observed events and discuss their relationship with the microwave solar radio bursts and with the observations of energetic electrons and protons ejected into space by the same flares. The central object of such a study is to reach a better understanding of the solar flare processes and the nature of the instability which generates flares but, in particular, to understand the processes which gave rise to suprathermal particles so frequently observed from flares. 
The X-rays are detected by ionization chambers carried for long periods of time outside the magnetosphere by the OGO-I and OGO-III satellites. Details of the instrumentation and previous work on this program have been summarized in several publications (Kane et al., 1966; Arnoldy et al., 1967a,b). The range of energies covered by the present experiments is similar to, but in general somewhat less than, the X-ray events detected previously by balloons flown near the top of the atmosphere (Peterson and Winckler, 1959; Winckler et al., 1961; Vette and Casal, 1961 ; Anderson and Winckler, 1962; Hofmann and Winckler, 1963). Several summaries of this very energetic bremsstrahlung emission from flares and their relationship to the radio and optical features are available, based on the older results (Winckler, 1963; Friedman, 1964; Kundu, 1965). In a general sense the energetic flare X-rays of energy above $10 \mathrm{KeV}$ appear as bursts of duration between 1 and $20 \mathrm{~min}$ in very good time simultaneity with the 'explosive' phase of flares (Moreton, 1964). Previously, the observations of these energetic X-rays were made by chance on high-altitude balloons carrying ion chambers or scintillation counters. These early experiments detected only the most energetic quanta from the flare due to the atmospheric absorption above the balloons. These rather exceptional events have given rise to considerable speculation about processes which could produce energetic quanta, such as the inverse Compton process (Acton, 1964; Shklovsky, 1964, 1965; Zheleznyakov, 1965), the synchrotron process (Stein and Ney, 1963), or nuclear processes giving gamma-rays. The results of the present study show that flares of all sizes from Class $1 \mathrm{~S}$ to Class 3B emit such energetic X-rays and that their origin is probably bremsstrahlung following the suprathermal heating of electrons in the magnetic-plasma medium in the solar active region. Our recent investigation (Arnoldy et al., 1967b) has shown that the X-ray bursts are well-correlated with the direct observations immediately afterward in space of energetic electrons greater than $40 \mathrm{KeV}$ energy which may well come from the same source (Lin and Anderson, 1967). In this paper and the previous related accounts we also have found evidence that the acceleration of solar flare protons which are now widely observed in interplanetary space or at the Earth may arise from a process disjoint from that responsible for the X-ray electron emission.

\section{Discussion of the OGO Experiments}

The X-rays were detected with an $18-\mathrm{cm}$ diameter aluminum wall ionization chamber filled with argon gas at $3.5 \mathrm{~atm}$ pressure. Identical instruments were provided for the OGO-I (launched September, 1964) and the OGO-III (launched June, 1966) satellites, both of which continue to give data. Details of the instrumentation are given in our previous publications (Kane et al., 1966; Arnoldy et al., 1967b). The ion-chamber response has a lower limit of $10 \mathrm{KeV}$, has a maximum response between 20 and $30 \mathrm{KeV}$ and an effective upper limit between 50 and $100 \mathrm{KeV}$ depending on the type of spectrum characteristic of the X-rays. In this paper the ion-chamber rates 


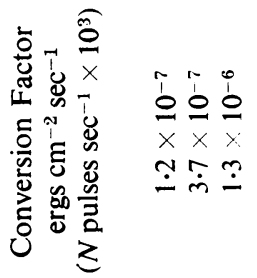

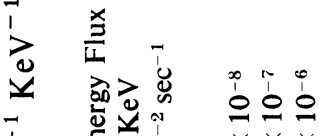

$$
\begin{aligned}
& \text { i) }
\end{aligned}
$$

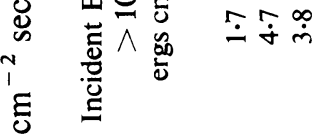

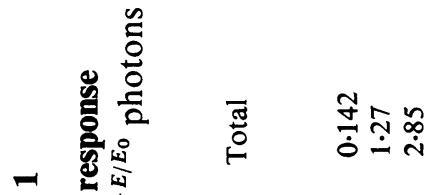

$$
\begin{aligned}
& \text { 尊 焉 }
\end{aligned}
$$

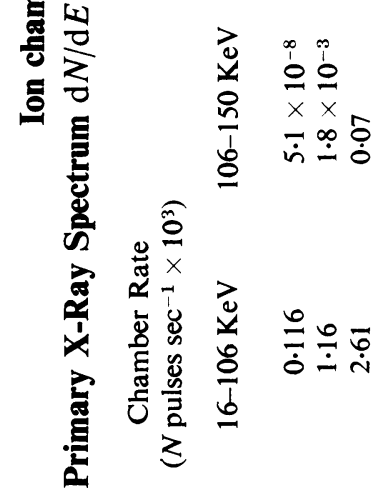

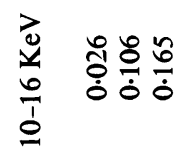

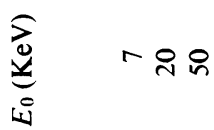


are given in the various figures in terms of a standardized arbitrary rate designated as normalized pulses per $\sec \times 10^{3}\left(\mathrm{NPPS} \times 10^{3}\right)$. The response characteristics and factors for converting the chamber rate to absolute energy flux are given in Table 1. For the analysis in this paper, we have assumed a rather steep exponential type X-ray spectrum with $E_{0}=7 \mathrm{KeV}$. The threshold sensitivity of the chamber for X-rays is $3 \times 10^{-7} \mathrm{ergs} \mathrm{cm}^{-2} \mathrm{sec}^{-1}$. The chamber responds also to protons above $12 \mathrm{MeV}$, to electrons above $700 \mathrm{KeV}$, and frequently detects particle events in space closely following the X-ray bursts from the same flare.

Recently some solar X-ray events have been detected simultaneously by both

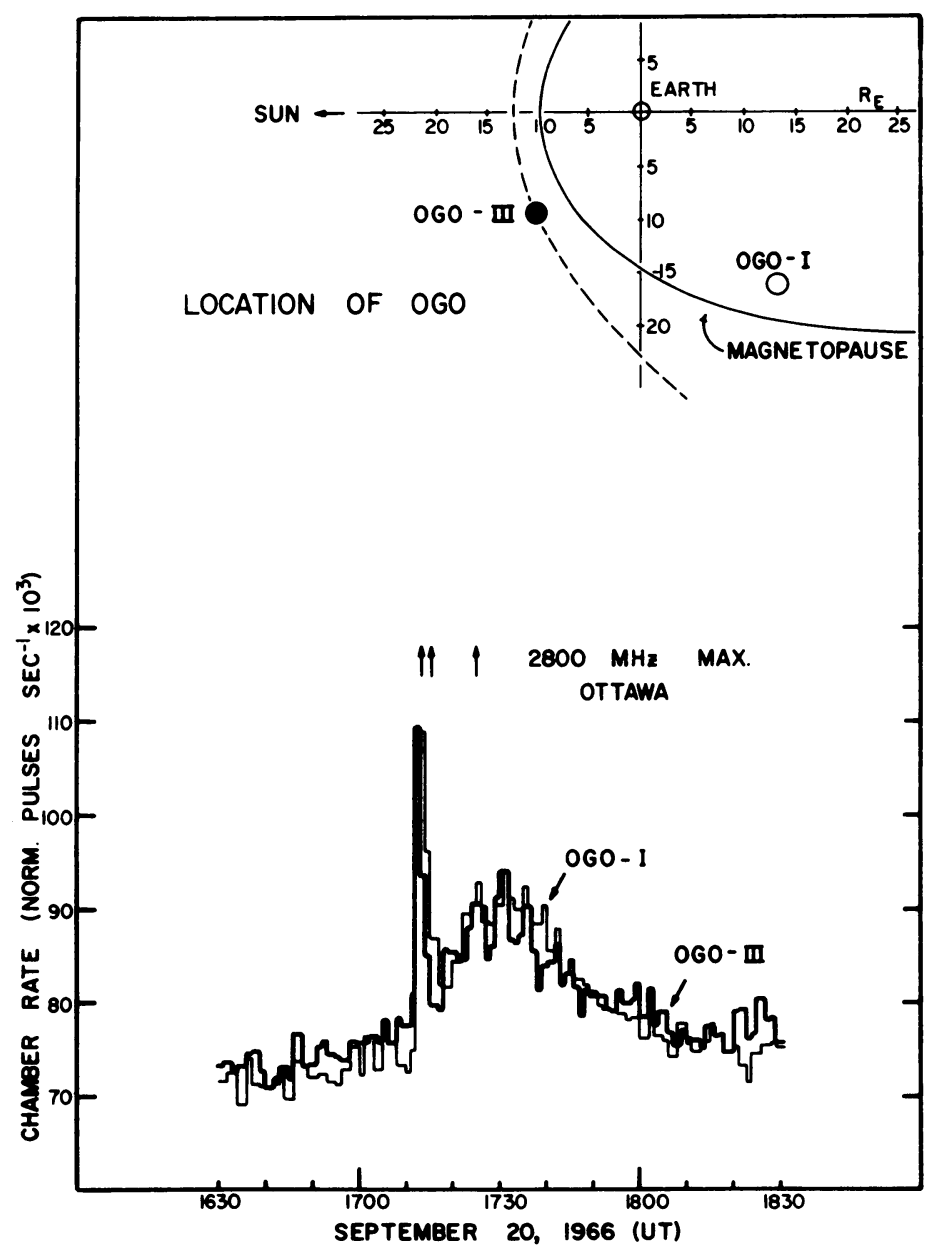

FIG. 1. A solar flare $X$-ray increase observed simultaneously by the ion chamber on OGO-I and OGO-III satellites. This X-ray burst has a sharp preliminary peak at 1712 UT and a broad maximum centered at 1730. A satisfactory $10-\mathrm{cm}$ radio correlation exists. 
OGO-I and III. Figure 1 shows such an example and also delineates the orbital positions of the two satellites with respect to the magnetospheric structure. Such simultaneous events make the identification of the X-ray increase completely certain and help to distinguish spurious cases due to electron bursts associated with the magnetosphere. The example shown in Figure 1 is also interesting because of the sharp burst at 1712 UT and the rather smooth maximum at 1730. The close agreement of the two ion chambers measuring the same event shows that the calibrations used were correct and that no drift has occurred in the calibration of the OGO-I instrument over a period of $2 \frac{1}{2}$ years.

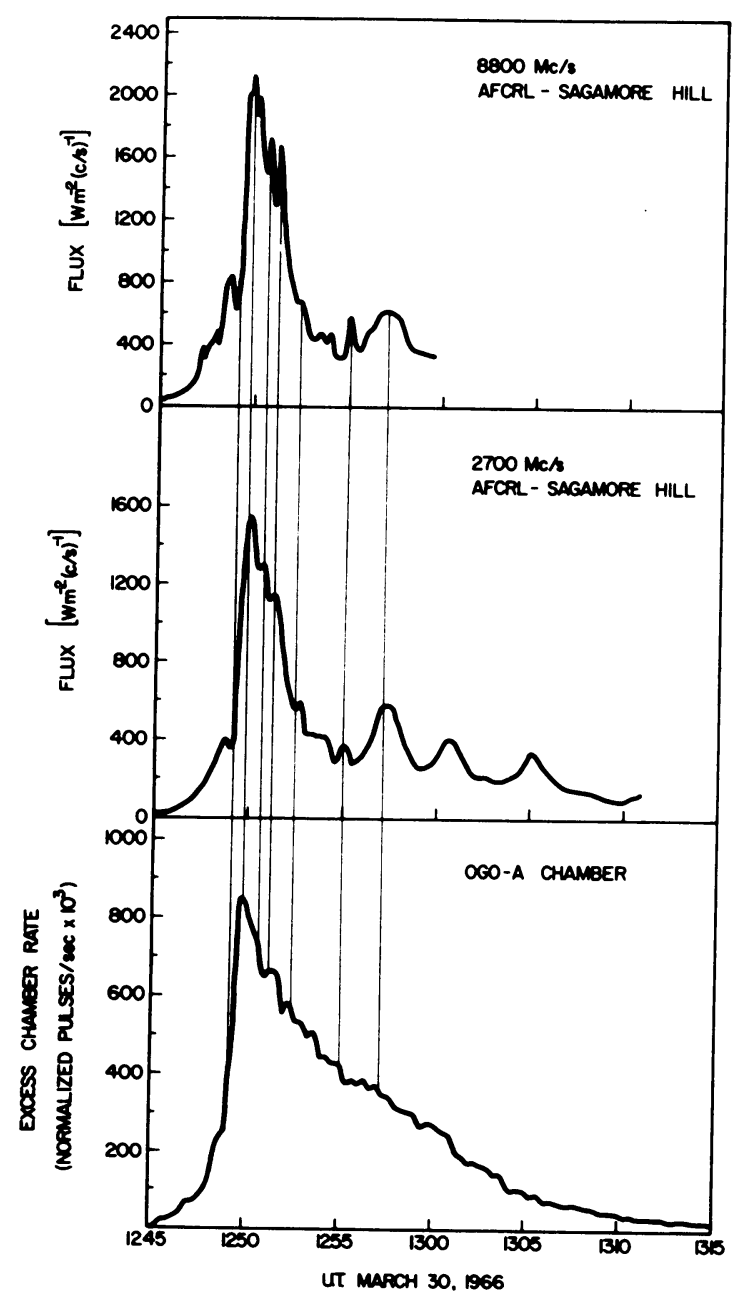

FIG. 2. Comparison of X-ray intensities (lower) and 3 and 10-cm radio emission (above) for a large complex flare event. 


\section{Correlation of X-Ray and Radio Bursts}

In Figure 2 we have a more or less typical large flare event producing a considerable intensity of energetic $\mathrm{X}$-rays and radio emission in the microwave range. We note a close similarity in the structure and rather good correlation of the start and time of maximum intensity. However, the fluctuations in the radio emission are much larger and do not always correspond to fluctuations in the X-ray intensity, which is observed in general to be much smoother. The decay of the March 30 event shown in Figure 2 is given in Figure 3, and one notes that it is roughly exponential but with the previously noted fluctuations in the radio emission. Approximately 70 events have now been analyzed constituting most of the cases observed up to December 31, 1966. The

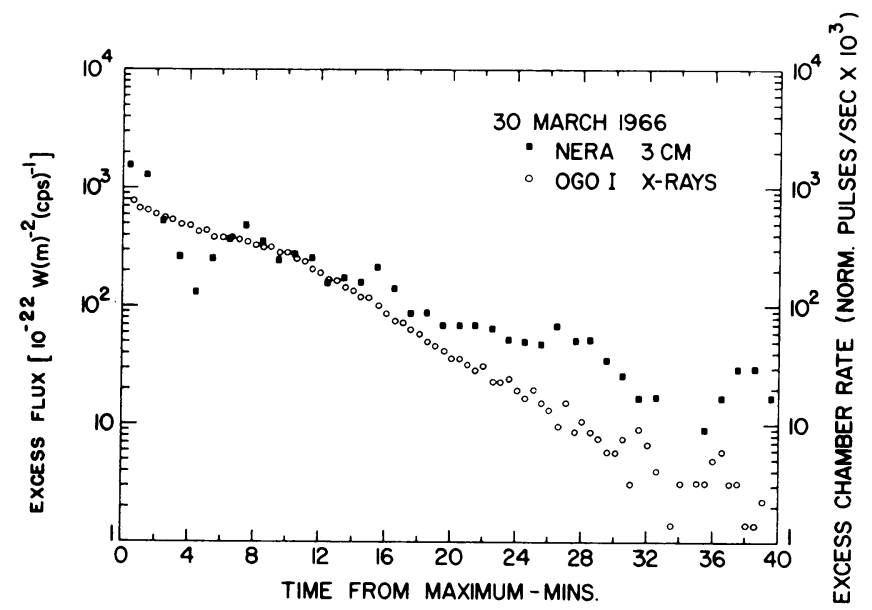

FIG. 3. The decay of a complex event showing similar trends for X-rays and 3-cm radio emission.

correlation plot of the integrated radio and X-ray flux is shown in Figure 4. The data are separated into two periods representing events prior to June 30, 1966 and events from July 1 to December 31, 1966, although a reasonable line may be constructed for the earlier period. With the advance of the solar cycle, there seems to be a systematic effect such that relatively more X-ray emission occurs with the same radio emission. Also, the scatter increases considerably especially for lower values of the fluxes. The solar cycle trend shown in this graph may possibly be associated with the appearance in the second half of 1966 of many centers of activity and sunspot zones in both solar hemispheres.

\section{Interpretive Remarks about the X-Ray Radio Relationship}

Because of the very close morphological relation between the X-ray production and 


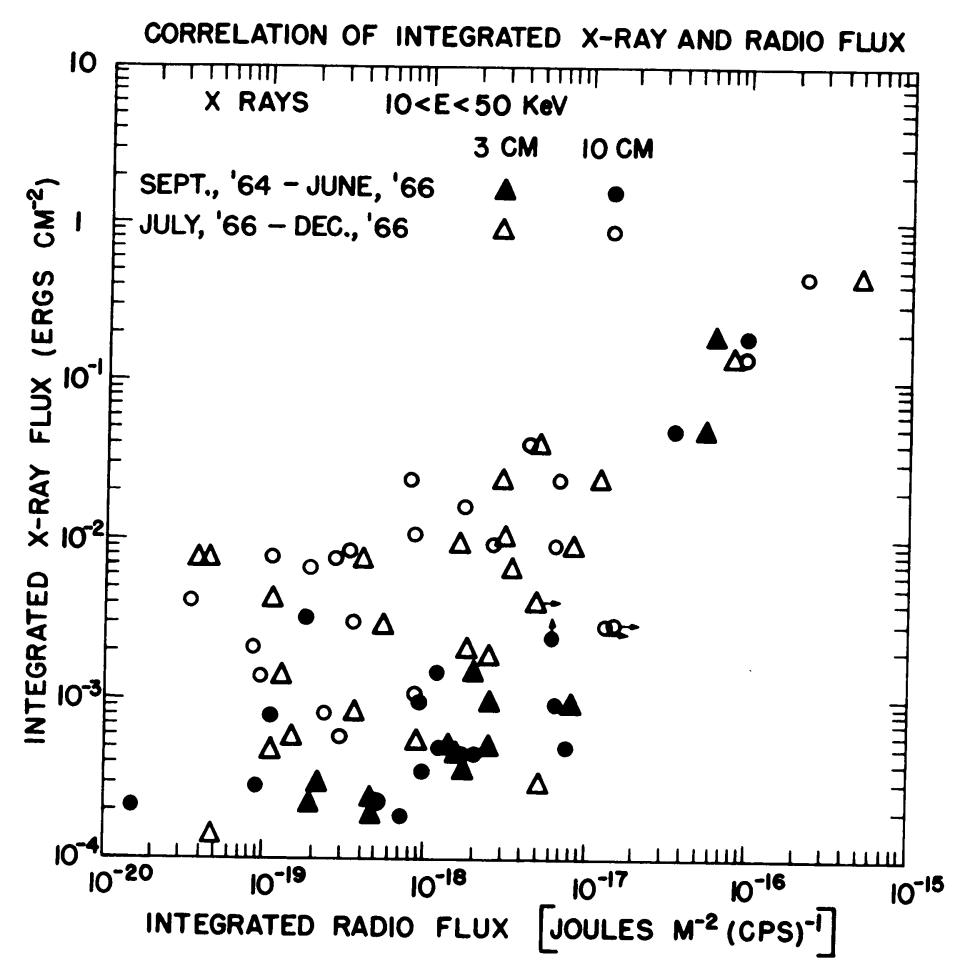

FIG. 4. Correlation of integrated $X$-ray and integrated radio flux for the observed events. The integral has been carried out over the first event in a flare occurrence. A roughly linear relation exists, but the events later in the solar cycle between July 1966 and December 1966 appear relatively more rich in X-ray emission than the earlier events.

the centrimetric range of radio emission it is very plausible to search for a model or to propose a situation in which both types of electromagnetic emission come from a common source. In the paper of Peterson and Winckler (1959) the source of the $\mathrm{X}$-rays was assumed to be bremsstrahlung from energetic electrons in an energy range around $500 \mathrm{KeV}$. The radio emission was then assumed to be by the synchrotron process from the same electrons. This lead to the difficulty that about $10^{4}$ times too much radio emission was expected compared to the observed. Takakura $(1963,1966)$ examined the situation and proposed that the region of emission was different for the $\mathrm{X}$-rays and the radio bursts and was able to adjust the radio power at the same time retaining the concept that the same energy region of electrons was responsible for both emissions.

The examples given in this paper have been presented purposely to show the complex character of the situation. Early in the flare event there appears to be a very close relationship between $\mathrm{X}$-rays and microwaves and as time progresses the radio emission assumes a time structure frequently not closely related to the smoothly disappearing 
X-ray burst. It will thus be difficult or impossible to form a single model applicable to all events. However, for the initial part of the events where the correlation is very strong between X-rays and microwaves, it may be possible. In our previous paper (Arnoldy et al., 1967b) we attempted to visualize the simplest possible situation that was consistent with the experimental facts and did not assume special kinds of processes not specifically dictated by the observations. We recall the approximate proportionality between the X-ray and radio emission as shown above and the similarity in duration and decay rate of the two types of emission. Two possible cases are considered: (a) that the characteristic time constant is determined entirely by the time variation of the basic energy source itself with all other time constants associated with specific processes (for example, synchrotron emission) being shorter than this. In case (b) the source is impulsive but a single dominant electron-decay process determines the time constant for both the $\mathrm{X}$-ray and radio emission. This time constant could be that required for the suprathermal electrons responsible for the bremsstrahlung to disappear by collision loss in the plasma. We consider that the same plasma region and probably the same electrons are producing both the X-ray and radio emission. The plasma is entrained in a magnetic field above the solar active centre. These fields must play a major role in the acceleration of particles and in the emission and propagation of radio-frequency energy. For any quantitative calculations the emission process must be made specific. Our model assumes that very hot energetic or suprathermal electrons lose energy predominantly by collisions with a much cooler plasma. This point of view has also been suggested by Elwert (1961). Neither the present measurements, nor the original measurements of Peterson and Winckler (1959) can provide exact energy discrimination. Thus a re-interpretation of the March 20, 1958 event of Peterson and Winckler, made by Chubb et al. (1966) in terms of an exponential spectrum with $E_{0}$ about $60 \mathrm{KeV}$ is probably acceptable. However, balloon scintillation counter measurements by Anderson and Winckler (1962) showed directly the presence of photons $>150 \mathrm{KeV}$ energy, and Cline et al. (1967) have shown flare bremsstrahlung spectra at $100 \mathrm{KeV}$. We do not consider plausible the concept that there exists a complete plasma at an enormously elevated temperature and that one is justified in using the thermal bremsstrahlung approach for computing the X-ray emission power for such events. The thermal bremsstrahlung approach with very high temperatures has been consistently proposed by the NRL group (see e.g. Chubb et al., 1966). It is true that the concept of temperature is often applied to one component of a medium, e.g. electron energies are frequently given in terms of electron temperature if the energy distribution appears to be exponential. However, one might expect that use of the concept of temperature would imply that the medium is close to a thermodynamic equilibrium condition and that one is therefore justified in applying this same temperature to compute many types of processes such as, e.g., the distribution of ionization states, spectral emission, etc. There appears to be no evidence at all that the temperatures deduced from other means in flare regions reach 
the enormous values of $10^{8 \circ}$ which one is forced to assume in order to produce the hard X-rays by thermal bremsstrahlung. Optical measurements (De Jager, 1959) frequently show $10000-20000^{\circ}$ as typical. We therefore favor the point of view that the electron heating in the flare region is highly non-equilibrium and may be associated with such phenomena as magneto-hydrodynamic waves and that there exists an energy distribution characteristic of a much lower temperature with a large suprathermal tail.

We now consider a quantitative estimate of the bremsstrahlung emission. In case (a), where the time behavior of the event is determined entirely by the energy source, the collision lifetime is very short. One can use the thick target bresmsstrahlung equation given by Koch and Motz (1959) for non-relativistic electrons

$$
\varepsilon=5 \times 10^{-4} Z \frac{E_{\mathrm{K}}}{m_{0} c},
$$

where $\varepsilon$ is the efficiency defined as (total energy radiated)/(total beam energy), $E_{\mathrm{K}}$ the kinetic energy, and $Z$ the atomic number of the target. Considering $100-\mathrm{KeV}$ electrons for a large event such as March 30, 1966 or July 7, 1966 a beam energy of $3 \times 10^{30}$ ergs is required, which is equivalent to $2 \times 10^{32}$ electrons. If we consider case (b), where an impulsive injection of $100-\mathrm{KeV}$ electrons is assumed the collision loss determines the decay of the event (from 1 to $10 \mathrm{~min}$ ) and gives density estimates of $3 \times 10^{10}$ atoms $\mathrm{cm}^{-3}$ to $3 \times 10^{9}$ atoms $\mathrm{cm}^{-3}$ for the lifetime range of 1 to $10 \mathrm{~min}$ respectively. The bremsstrahlung power is calculated also from the results of Koch and Motz (1959) valid for electron energies of $10-100 \mathrm{KeV}$ incident on neutral hydrogen. Quantitatively, the relationship reduces to the following, where $P$ is the number of photons between 10 and $50 \mathrm{KeV}$ per $\mathrm{cm}^{2}$ per sec measured at the Earth, and $N_{\mathrm{H}}$ and $N_{\mathrm{e}}$ are respectively the densities of hydrogen and energetic electrons situated in the volume $V$.

$$
P=3 \times 10^{-43} N_{\mathrm{H}} N_{\mathrm{e}} V \text { photons } \mathrm{cm}^{-2} \mathrm{sec}^{-1} .
$$

If $N_{\mathrm{H}}$ can be estimated from observed event lifetimes, and if $P$ is measured, then Equation (2) may be used to compute the total number of electrons, $N_{\mathrm{e}} V$. Again for a large X-ray event the value of $P$ is $1.5 \times 10^{4}$ and from the observed mean lifetime of $300 \mathrm{sec} N_{\mathrm{H}}$ is estimated to be $5 \times 10^{9} \mathrm{~cm}^{-3}$. For this density $10^{37}$ electrons are required in the impulsive injection process, i.e. essentially the same number as calculated under assumption (a).

It is necessary to point out that in the thick target case (a) we begin with $100 \mathrm{KeV}$ electrons but the energy is degraded to zero in the thick target as they are brought to rest. Thus the emission occurs for all energies between 0 and $100 \mathrm{KeV}$. A portion of this emitted spectrum is not detected by the ionization chamber which results in an error of probably not more than a factor of 2 in applying the Koch and Motz thick target equation. In case (a) the integrated bremsstrahlung energy over the entire observed X-ray burst is used for the comparison. In case (b) we utilize the Koch and Motz thin target approximation for electrons of the unique energy of $100 \mathrm{KeV}$ equal 
to the assumed injection energy. For comparison with the theory therefore the measured peak X-ray burst intensity is used rather than the integrated intensity.

Considering the radio emission this is often attributed to synchrotron radiation. Although this is certainly a plausible mechanism the detailed measurements of polarization and other factors do not exclude a thermal source accompanied by propagation effects which produce polarization. In fact, Kundu (1965) suggests that the microwaveburst events may frequently be a mixture of thermal and synchrotron emission. Considering a large event such as March 30, 1966 and July 7, 1966 the microwave

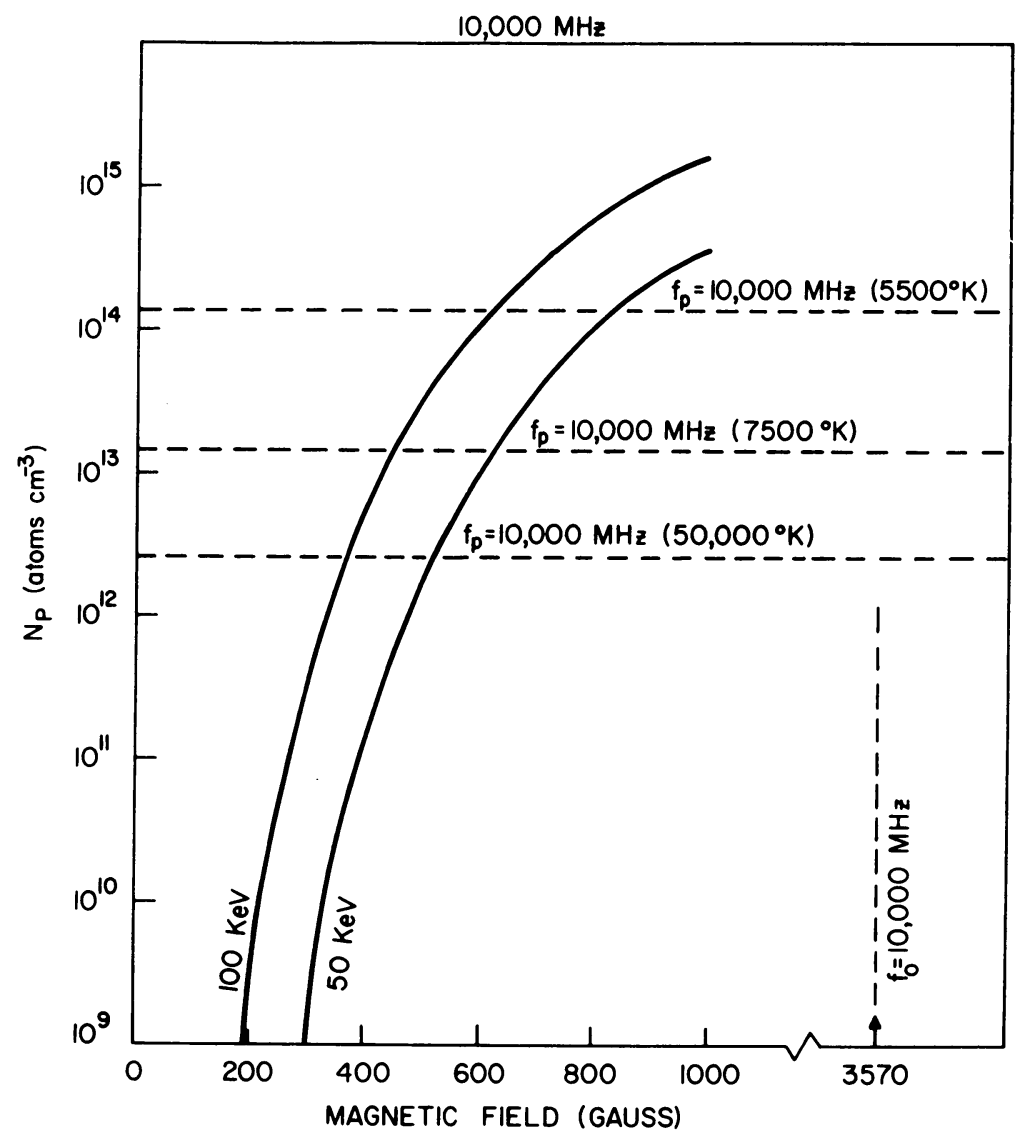

FIG. 5. The density required to give the observed bremsstrahlung power per electron expressed as a function of the magnetic field required to give the observed radio emission at $10000 \mathrm{MHz}$ by the synchrotron process. The relation is shown for discrete energies of $50 \mathrm{KeV}$ and $100 \mathrm{KeV}$. Any point along the curves will satisfy the observed proportionality between the microwave and $X$-ray emission under the assumptions presented in the text. The densities at which the plasma frequency of the medium is equal to $10000 \mathrm{MHz}$ is indicated for various temperatures. The magnetic-field strength which gives an electron gyro-frequency of $10000 \mathrm{MHz}$ is also shown. 
energy received at earth at $3 \mathrm{~cm}$ was approximately $6 \times 10^{-17}$ joules $\mathrm{m}^{-2}(\mathrm{CPS})^{-1}$. Following our basic assumption that the same electrons are responsible for both processes, about $5 \times 10^{-54}$ joules $\mathrm{m}^{-2}(\mathrm{CPS})^{-1}$ of microwave energy at the Earth are required per electron at the Sun on the average. This number will be approximately the same for all events as long as the proportionality between X-ray and microwave emission is valid. The large difference in size from event to event is thus principally due to the difference in number of electrons in the source.

For the purposes of discussion we have assumed that the microwave energy is from the synchrotron emission of electrons of intermediate energy. Following the work of Takakura (1960) for electrons of intermediate energy and assuming 50 and $100 \mathrm{KeV}$ energies one can calculate the total power radiated by an electron in uniform circular motion averaged over $4 \pi$ solid angle. For a given frequency and electron energy the power radiated per electron depends only on the magnetic field $B$. On the other hand, for bremsstrahlung the power radiated per electron depends only on the density of hydrogen. Thus, as shown in Figure 5, one achieves a relation between density and magnetic field for the two chosen discrete energies such that the observed proportionality between energetic X-ray and 3-cm emission is satisfied. In case (b) (impulsive injection by source) it becomes apparent that the $3-\mathrm{cm}$ emission is occurring close to the 20th harmonic for an acceptable magnetic field. The emissivity at this harmonic is several orders of magnitude below that near the gyrofrequency and we consider this to be a very improbable physical situation. However, if one allows higher densities and very short electron lifetimes as in case (a) (where the duration of the event is controlled by the source) then the permissible magnetic field is much higher and the harmonic is reduced to a plausible value. In practice one would, of course, wish to use a continuous spectrum of electron energies such as the computation of Takakura (1966). But with the very short lifetime due to collisions and a re-generation continuously during the event by the source one achieves with reasonable magnetic field strengths the low stored flux of electrons required to agree with the observed radio emission. Thus the original difficulty in the calculation of Peterson and Winckler is resolved.

Figure 5 shows also the density corresponding to the plasma frequency for the ionized component at several temperatures and also on the $x$-axis with an extended scale the magnetic field corresponding to the gyro-frequency. The radio emission must occur within the rectangle limited by these lines.

The relationship shown in Figure 5 between density and magnetic-field strength corresponding to the discrete energies $50 \mathrm{KeV}$ and $100 \mathrm{KeV}$ may possibly define some direct physical situation in the flare region. We show in Figure 6 a mean curve for $75 \mathrm{KeV}$ and a family of curves for different values of the ratio, $\beta$, of thermal energy to magnetic energy for a chosen temperature of $7500^{\circ} \mathrm{K}$. One might suppose that during an active time the magnetic field above the sunspot regions would be carrying a large plasma density and that possibly the $\beta$-value might tend to be constant in 
different portions of the field. However, the empirical curve for $75 \mathrm{KeV}$ spans several orders of magnitude variation in $\beta$, as shown in Figure 6. If, indeed, there is any direct physical significance to this curve then it implies that the magnetic field is more heavily loaded with plasma for high field strengths and very lightly loaded in the upper corona where the field strengths are small.

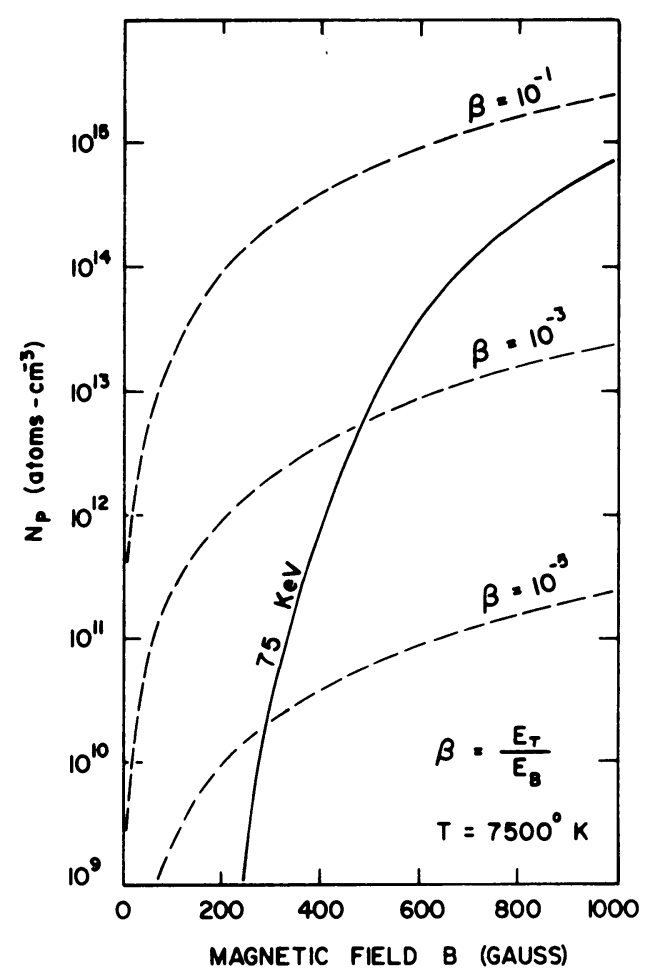

FIG. 6. An examination of the hypothesis that the magnetic-field density curve (for mean energy $75 \mathrm{KeV}$ ) physically represents the thermal to magnetic-energy relationship in the plasma.

The discussion above may be considered applicable to the 'first' event in a flare where a good correlation between $\mathrm{X}$-ray and radio emission is observed. The complex character of the $X$-ray radio relationship is shown by many events. Figure 7 shows data from various sources relating to the large flare on July 7,1966 . Besides the radio emission and the OGO ionization chamber record, a high-energy scintillation counter also on the OGO satellite (Cline et al., 1967) is plotted. We note that the $25 \mathrm{KeV}$ range of X-rays again is similar in its structure and time duration to the radio emission. The $>80 \mathrm{KeV}$ X-rays, however, show a much shorter time constant reminiscent of the older events observed by balloons. However, the maximum intensity in all ranges seems to occur at closely the same time. 
Another event representative of many already observed (Figure 8) shows that the energetic X-ray emission is correlated closely with centimetric range of frequencies and that the appearance later in the event of a spectrum rich in the metric region does not correlate with X-ray emission. This may be interpreted to show that the production region of the X-rays is deep in the chromosphere and that the electrons responsible for the metric wave emission are at greater heights in the corona.

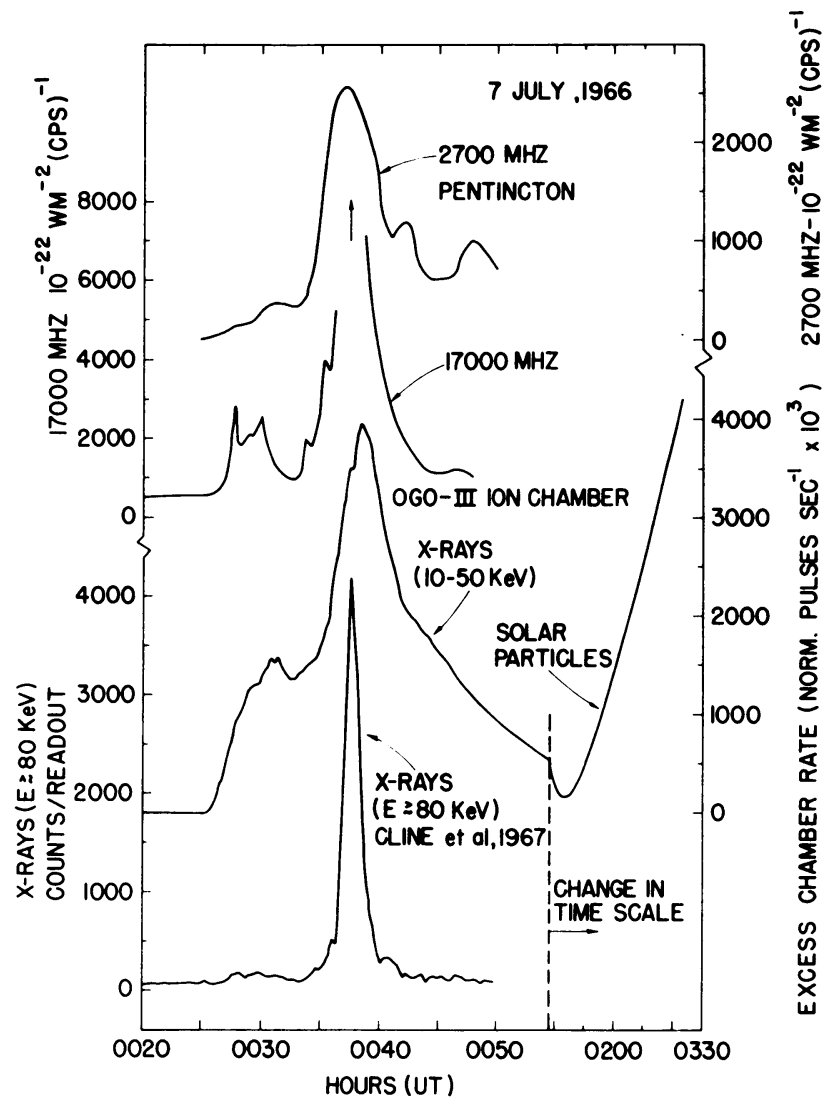

FIG. 7. Radio and hard X-ray emission for the flare of July 7, 1966. Note that while the $25 \mathrm{KeV}$ $X$-ray range shows a time structure similar in duration to the microwave emission, the $>80 \mathrm{KeV} \mathrm{X-ray}$ emission is very short, like cases observed earlier by balloon equipment.

In the inverse sense sometimes double X-ray bursts occur for which the second is not well correlated with a maximum in the radio emission. Such a case is shown in Figure 9. In Figure 10 we see again an event observed earlier by balloon apparatus (Hofmann and Winckler, 1963) in which there is a large double microwave event but for which only the first part produces a detectable X-ray increase. This particular 
event on July 20, 1961 has been widely studied (Bruzek, 1964; Ellison et al., 1961) and occurred on the solar limb.

\section{Energetic Particle Relationships}

The OGO ionization chamber can detect solar particle events as well as X-ray bursts and frequently shows intensity increases following a flare which are clearly due

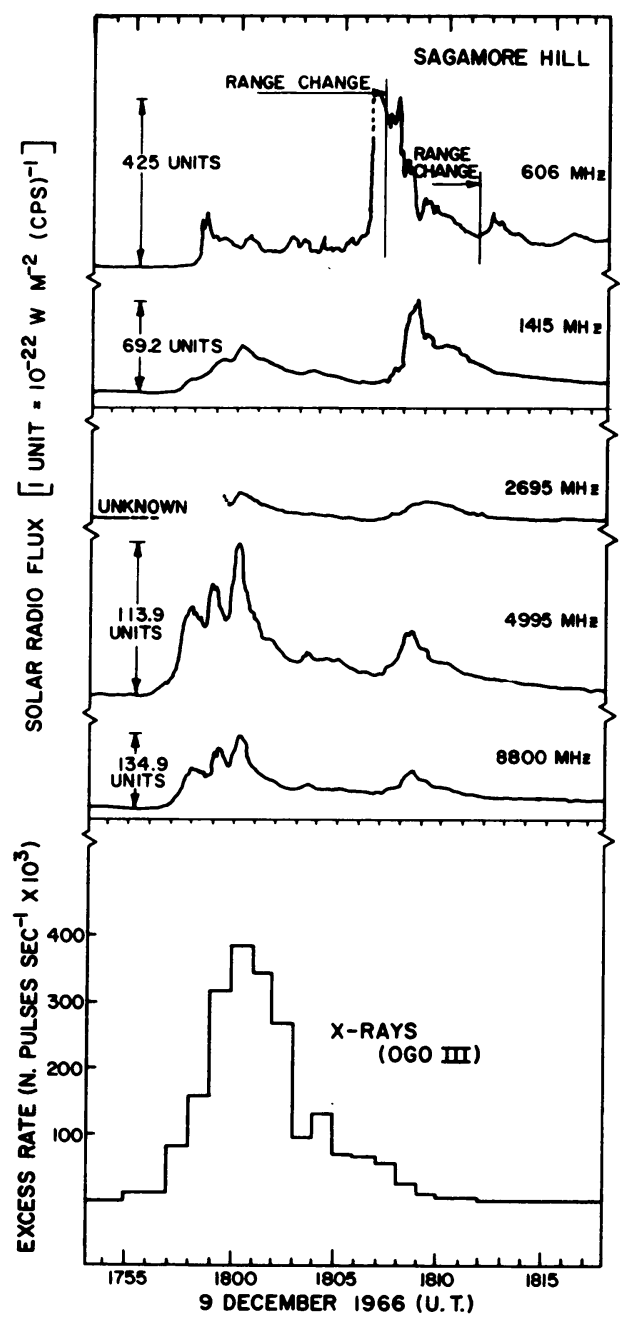

FIG. 8. An example of a good $X$-ray radio correspondence for the first event in the flare beginning at 1757 but showing the lack of observable X-rays corresponding to the predominantly metric event beginning at 1806. 


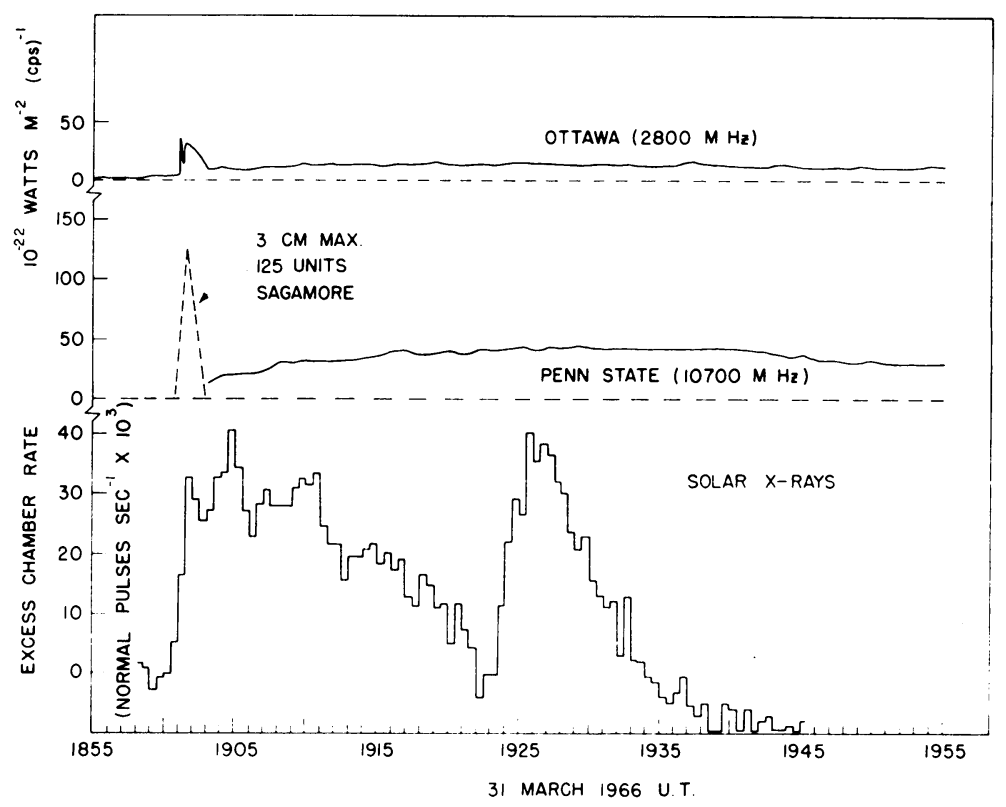

FIG. 9. A large double $X$-ray event corresponding to a long-duration microwave increase. No detectable radio event corresponds to the second X-ray maximum at 1927 UT.

to charged particles from the Sun. The ionization chamber is very sensitive to protons and can detect a flux of 0.01 protons $\mathrm{cm}^{-2} \mathrm{sec}^{-1}$ of energy greater than $12 \mathrm{MeV}$. The chamber also is sensitive to electrons greater than $700 \mathrm{KeV}$ but in principle cannot distinguish the type of particle responsible for a given increase. An example of a small particle event following an X-ray event on June 5, 1965 is shown in Figure 11. The event begins at 1900 UT and reaches a maximum at 2100 UT. Interpreted as protons, this implies 0.07 protons $\mathrm{cm}^{-2} \mathrm{sec}^{-1}$ but this increase could also presumably be due to electrons greater than $700 \mathrm{KeV}$. In fact, this event produced an identifiedelectron increase in both the Mariner-IV and IMP-I satellites (Van Allen and Krimigis, 1965; Lin and Anderson, 1967) of energies greater than $40 \mathrm{KeV}$.

A very large X-ray event on August 28, 1966 followed by a solar proton event which eventually became very large is shown in Figure 12 . In this case the X-ray increase was roll modulated by the spinning spacecraft when the ion chamber was eclipsed by the body of the spacecraft. One notes the lack of roll modulation for the particle event. The maximum in solar particle intensity was reached at 2100 UT with an ion-chamber rate of 15000 NPPS $\times 10^{3}$. One notes the lack of correlation between the large radio maximum at 1605 and the X-ray intensity.

The release into space of electrons above $40 \mathrm{KeV}$ energy associated with solar flares has now been observed on many occasions by the IMP spacecraft (Lin and Anderson, 
1967). It is possible to use IMP data to determine which OGO particle events did not contain electrons and presumably were due to solar protons. Also both the IMP electron events and the proton events can be correlated with the occurrence of energetic X-ray bursts and microwave emission. A complete summary with tables for the period September, 1964 through June, 1966 has been given in our previous paper (Arnoldy et al., 1967b). These correlations may be summarized as follows:

(a) Of 8 proton events observed by the ionization chamber but not detected as electron events on IMP satellite, 7 were not associated with solar flare energetic

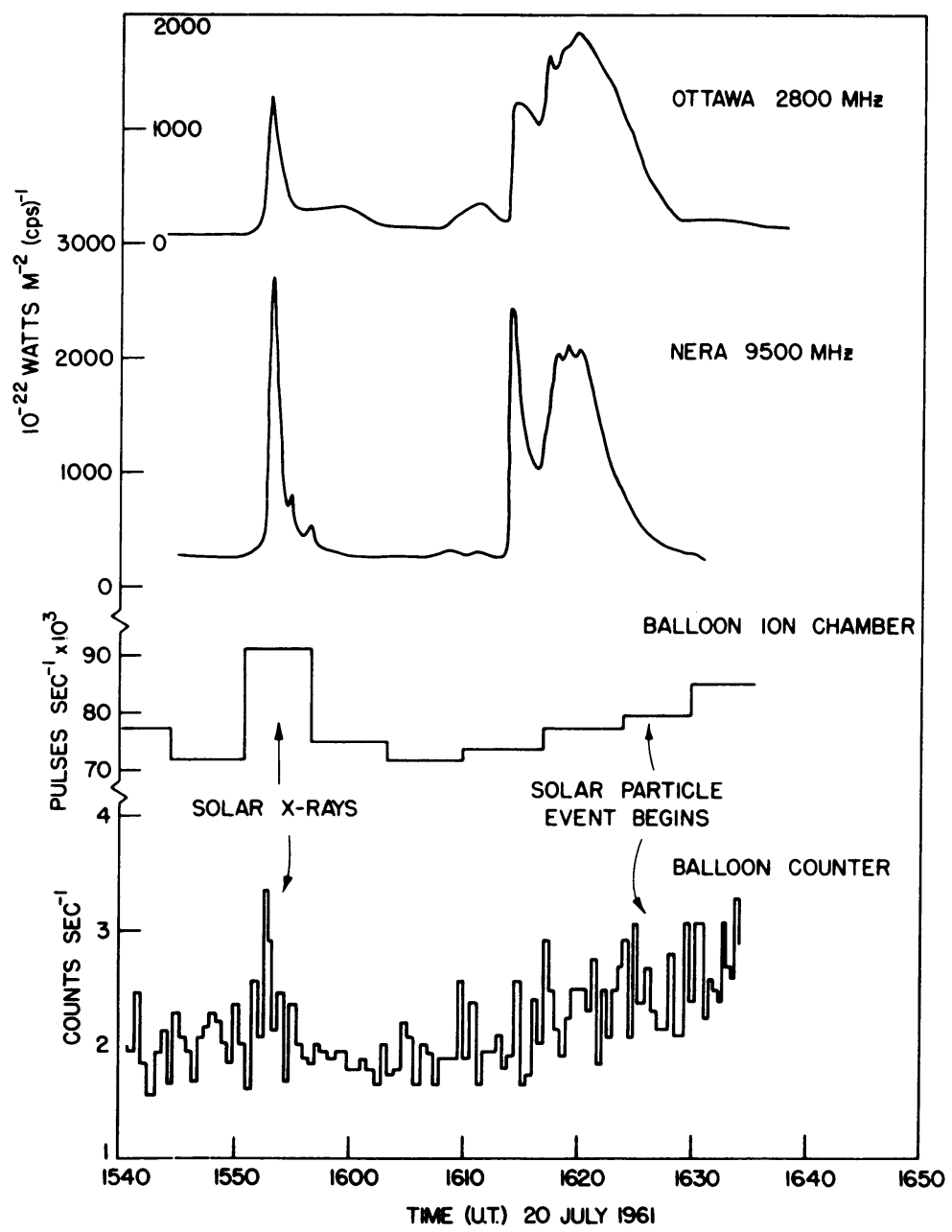

FIG. 10. An example of a limb event observed by balloon equipment in which the first increase produces a solar X-ray event but the second large microwave increase has no detectable X-rays. A solar proton increase from this West limb flare has begun by 1612. (See Hofmann and Winckler, 1963.) 
X-rays. An example of such an event was given as Figure 20 of our previous paper (Arnoldy et al., 1967b). It appears, therefore, that the production of solar protons is not necessarily closely correlated with the processes producing electrons and energetic bremsstrahlung.

(b) Eight large electron events detected when the OGO and IMP spacecraft were simultaneously operating in interplanetary space had associated X-ray bursts. The remaining 4 electron events were very small and were not recorded by OGO as X-rays

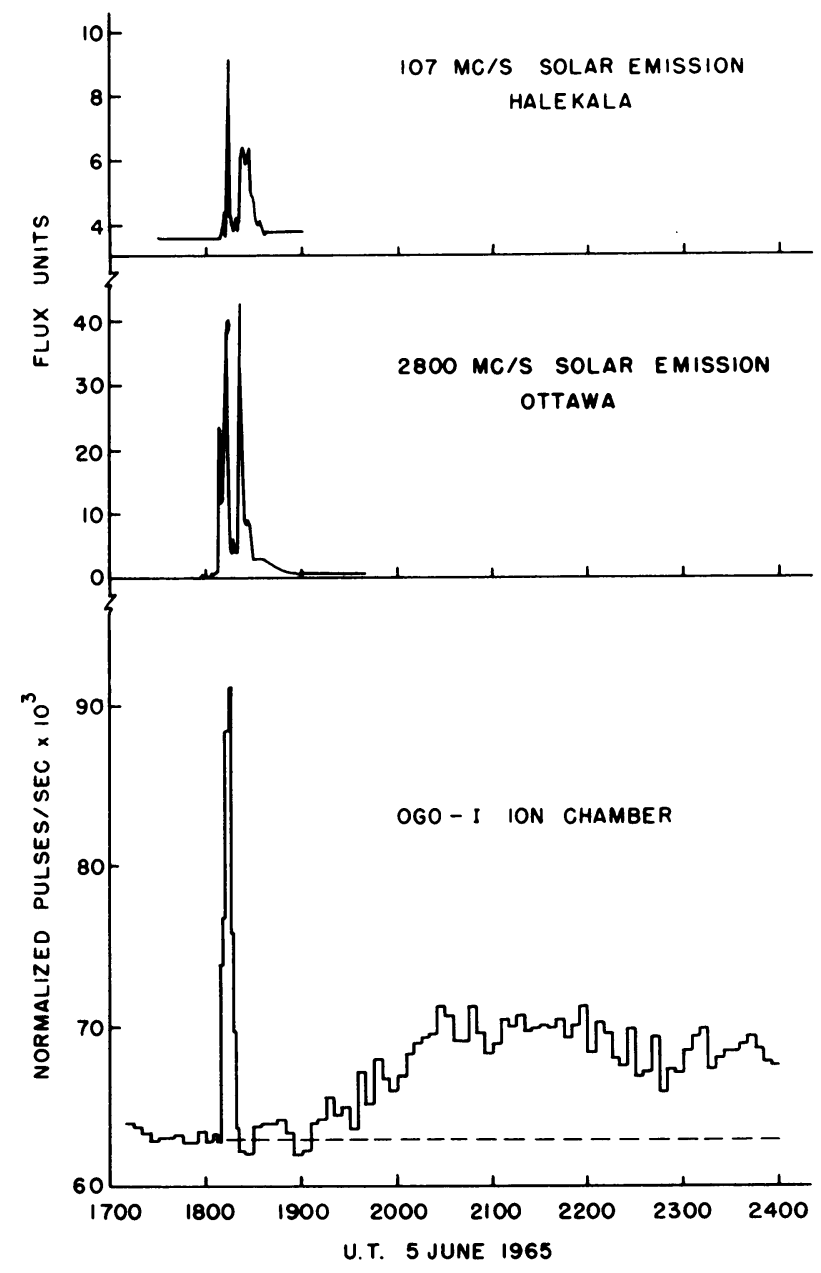

FIG. 11. A small X-ray event showing the ensuing solar particle event observed by the ionization chamber. These particles are not specifically identified by the OGO ion chamber but may be solar protons. Solar electrons were observed by several experiments (see text) and correspond to this particle increase. 
nor was there microwave emission. This rather good correlation of X-ray bursts with interplanetary electron events suggests that the flare electrons that leak out into interplanetary space might be from the same suprathermal source as those responsible for the energetic X-rays. For the June 5, 1965 event the observed number of electrons measured directly in space above $40 \mathrm{KeV}$ can be provided by the leakage into interplanetary space from the flare region of about $0 \cdot 1 \%$ of the number of electrons required to produce the corresponding X-ray event.

(c) The reverse correlation where one begins with known OGO X-ray bursts and compares IMP data for interplanetary electrons shows 12 events with no interplanetary electrons. It is striking that all 12 were produced by flares near or East of central meridian. It is very reasonable to assume that the absence of electrons in these cases is due to a propagation asymmetry between the Sun and the Earth similar

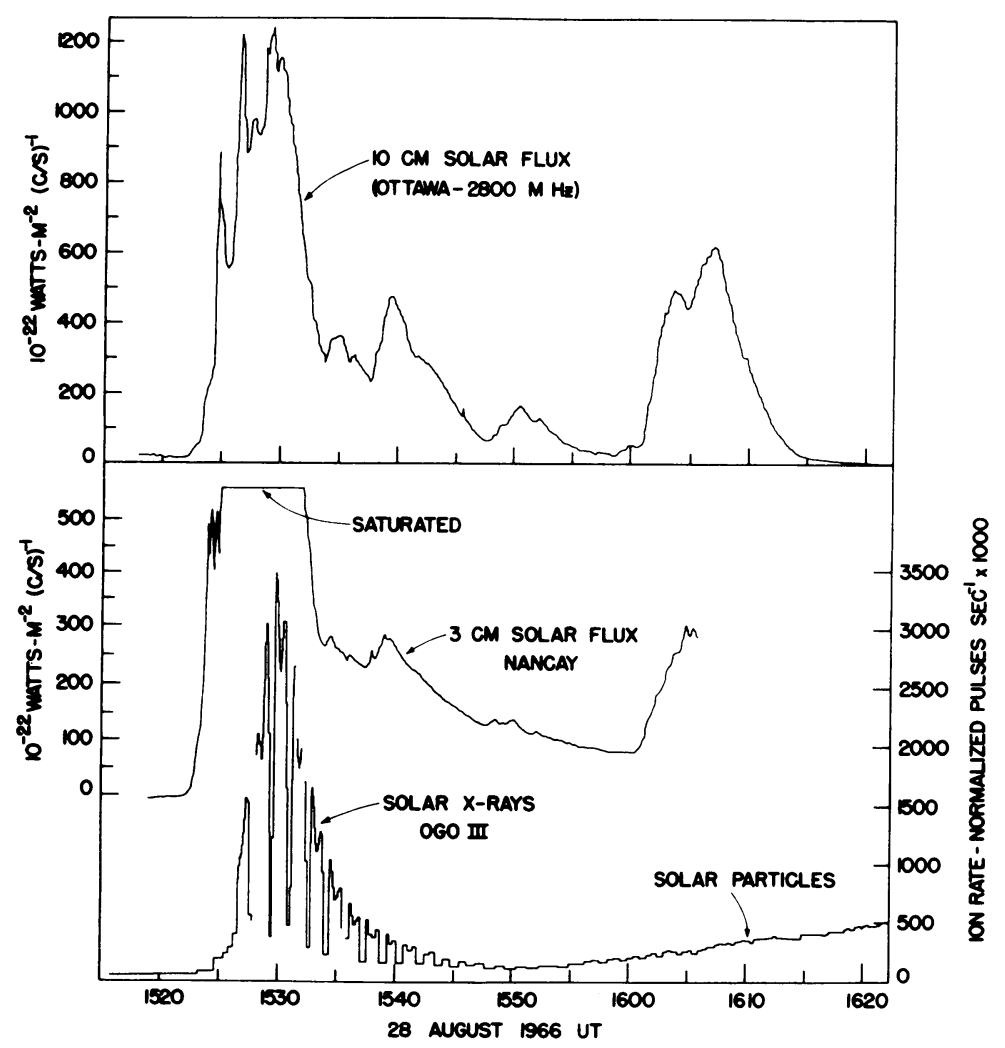

FIG. 12. The great event on August 28, 1966. Note the almost complete roll modulation of the $X$-ray burst with the 96-sec roll period of OGO-III. This roll modulation is not present in the solar particle increase beginning at 1550 UT. Only the initial features of the complex radio burst are reflected in the $X$-ray profile. 
to that previously observed for high-energy solar protons caused by the spiral interplanetary magnetic field.

(d) It is generally found that very large flares such as July 7, 1966 or August 28, 1966 produce all types of energetic solar phenomena simultaneously. X-rays, microwave emission, interplanetary electrons and solar protons are all observed.

\section{Acknowledgments}

We are indebted to the radio observatories of Penn State, Nancay, Nera, Toyokawa and Ottawa who have furnished many original records for this study and to Dorothy Trotter, World Data Center A for Solar Activity, for lists of outstanding occurrences. Preliminary use has been made of optical data prepared for us by Helen W. DodsonPrince and E. Ruth Hedeman of McMath-Hulbert Observatory. We are also grateful to Dr. M. Pick of Meudon Observatory for important assistance with solar radio and optical interpretation, to R. Lin and Professor K. A. Anderson of the University of California, Berkeley for assisting with the IMP electron correlations, and to Mr. Karl Pfitzer for major assistance with OGO-data reduction. Data analysis was assisted by a Guggenheim Fellowship to one of the authors (J.R. Winckler) for study at the Observatoire de Meudon. This work has been supported by the National Aeronautics and Space Administration under Contract NO. NAS5-2071.

\section{References}

Acton, L.W. (1964) Nature, 204, 64-65.

Anderson, K.A., Winckler, J.R. (1962) J. geophys. Res., 67, 4103-4117.

Arnoldy, R. L., Kane, S.R., Winckler, J.R. (1967a) Solar Phys., 2, 171.

Arnoldy, R.L., Kane, S.R., Winckler, J.R. (1967b) University of Minnesota Technical Report CR-97. Also, to be published in Astrophysical Journal, February, 1968.

Bruzek, A. (1964) AAS-NASA Symposium on the Physics of Solar Flares, Scientific and Technical Information Division, NASA, Washington, D.C., 301-322.

Chubb, T.A., Kreplin, R.W., Friedman, H. (1966) J.geophys. Res., 71, 3611-3622.

Cline, T.L., Holt, S.S., Hones, Jr., E.W. (1967) Goddard Tech. Report X-611-67-348, 1965 Cospar Symposium, London.

De Jager, C. (1959) in Handbuch der Physik, Springer-Verlag, Heidelberg, LII, p. 205.

Ellison, M.A., McKenna, S.P., Reid, J.H. (1961) Dunsink Obs. Publ., 1, 75.

Elwert, G. (1961) J. geophys. Res., 66, 391-401.

Friedman, H. (1964) AAS-NASA Symposium on the Physics of Solar Flares, Scientific and Technical Information Division, NASA, Washington, D.C., 147-157.

Hofmann, D.J., Winckler, J.R. (1963) J.geophys. Res., 68, 2067-2099.

Kane, S. R., Pfitzer, K.A., Winckler, J.R. (1966) University of Minnesota Technical Report CR-87. Koch, H.W., Motz, J.W. (1959) Reviews of Modern Physics, 31, 920-955.

Kundu, M.K. (1965) Solar Radio Astronomy, Interscience Publishers, New York, pp. 530-534. Lin, R.P., Anderson, K.A. (1967) Solar Phys., 1, 446-464.

Moreton, G.E. (1964) AAS-NASA Symposium on the Physics of Solar Flares, Scientific and Technical Information Division, NASA, Washington, D.C., 209-212.

Peterson, L.E., Winckler, J.R. (1959) J. geophys. Res., 64, 697-701.

Shklovsky, J. (1964) Nature, 202, 275. 
Shklovsky, I.S. (1965) Soviet Astr. - AJ, 8, 538-543.

Stein, W. A., Ney, E.P. (1963) J. geophys. Res., 68, 65-81.

Takakura, T. (1960) Publ. astr. Soc. Japan, 12, 326-346.

Takakura, T. (1963) private communication.

Takakura, T., Kai, K. (1966) Publ. astr. Soc. Japan, 18, 57-76.

Van Allen, J. A., Krimigis, S. M. (1965) J. geophys. Res., 70, 5737-5751.

Vette, J.I., Casal, F. G. (1961) Phys. Rev. Lett., 6, 334-336.

Winckler, J.R. (1963) AAS-NASA Symposium on the Physics of Solar Flares, Scientific and Technical Information Division, NASA, Washington, D.C., 117-127.

Winckler, J.R., May, T.C., Masley, A.J. (1961) J.geophys. Res., 66, 316.

Zheleznyakov, V.V.(1965) Soviet Physics - Astronomy, 9, 73-76. 